\title{
A comprehensive evaluation of an ELISA for the diagnosis of the two most common ascarids in chickens using plasma or egg yolks
}

Gürbüz Daş ${ }^{1 *}$, Mark Hennies ${ }^{2}$, Birgit Sohnrey ${ }^{3}$, Shayan Rahimian³ ${ }^{3}$ Kalyakorn Wongrak ${ }^{4}$, Manuel Stehr ${ }^{1}$ and Matthias Gauly ${ }^{5}$

\begin{abstract}
Background: Classical faecal egg counts (FEC) provide less reliable diagnostic information for nematode infections in chickens. We developed an ELISA based on Ascaridia galli antigens and tested two hypotheses, as follows: (i) IgY antibodies developed against $A$. galli will also be useful to identify Heterakis gallinarum infections, and (ii) circulating antibodies stored in egg yolks are as good as plasma samples, so a non-invasive diagnosis is possible. The aim of this study, therefore, was to compare the diagnostic accuracy of the ELISA system with FEC, using both plasma and egg yolks from experimentally infected hens. In addition, naturally infected animals were evaluated to validate the assay.

Results: The assay quantified large differences $(P<0.001)$ in plasma or in egg-yolk IgY concentrations between infected and uninfected animals in two experiments, each performed with either of the nematode species. The assay performed with high accuracy as quantified with the area under the ROC curve (AUC) values of $>0.90$ for both nematodes using either plasma or egg yolks. Sensitivity of the assay was 94 and 93\% with plasma and egg yolk samples, respectively, whereas FEC yielded in a sensitivity of $84 \%$ in A. galli experiment. Total test accuracy of the assay with plasma samples ( $A \cup C=0.99)$ tended to be higher $(P=0.0630)$ than FEC (AUC $=0.92)$ for $A$. galli, while the assay with either sample matrix performed similar to FEC (AUC $\geq 0.91$ ) for $\mathrm{H}$. gallinarum. Among the three tests, the FECs correlated better with $A$. galli burden than the ELISA. Although $90 \%$ of naturally infected hens were correctly identified by the ELISA, $45 \%$ of the infected hens tested negative with FEC, indicating the validity of the higher test accuracy of the ELISA.

Conclusions: Antigens of A. galli can be used successfully to identify H. gallinarum-infected animals, indicating that chickens develop cross-reactive antibodies against the two closely related species. Egg yolks are as informative as plasma samples, so that animal welfare-friendly sampling is possible. Although the assay with plasma samples reveals qualitative information of higher quality than FECs on the infection status of naturally infected birds, the latter is still a better tool to assess the intensity of $A$. galli but not of $H$. gallinarum infections.
\end{abstract}

Keywords: Helminth, Nematode, Non-invasive diagnosis, Poultry, ROC analysis, Test accuracy

\footnotetext{
* Correspondence: gdas@fbn-dummerstorf.de

'Institute of Nutritional Physiology 'Oskar Kellner', Leibniz Institute for Farm

Animal Biology, Wilhelm-Stahl-Allee 2, 18196 Dummerstorf, Germany

Full list of author information is available at the end of the article
} 


\section{Background}

Infections of chickens with gastrointestinal nematodes, particularly with the roundworms Ascaridia galli and Heterakis gallinarum, are re-emerging in European laying hen farms that operate with the obligatory non-cage housing systems [1-5]. Although a few other nematode species, e.g. Syngamus trachea and Capillaria spp., and a few cestode species are also encountered [2], the highest prevalence and worm burdens are from two phylogenetically closely related $[6,7]$ nematode species, $A$. galli and $H$. gallinarum. Both species are pathogenic to chickens $[8,9]$ and can impair overall productivity of hens through direct and indirect effects. Direct effects result from the pathogenic consequences of infections, e.g. damage to the intestinal tissue [10] and resulting impairments in overall nutrient absorption, utilization and growth [11-13] as well as mortality, which may be caused by the obstruction of intestines in heavy infections with $A$. galli [14], although recent evidence also indicates an association between the presence of both nematode infections and hen mortality [15]. Vectoring roles for other parasites (e.g. Histomonas meleagridis by H. gallinarum [16]) or bacteria (e.g. Salmonella enterica by A. galli [17]) and the impaired humoral responses after vaccinations against other pathogens (e.g. Newcastle disease virus) [18] may be considered as the most important indirect effects of the infections. Moreover, animal welfare, which was expected to improve with the EU legislative ban on battery cages [19], is also threatened/endangered because of the overall effects of the infections on animal health and welfare [20].

The occurrence, and in some cases, the intensity of nematode infections in living chicken hosts are classically determined by faecal egg counts (FEC). However, obtaining suitable individual faecal samples from the chicken host is problematic because of the small host size, which does not allow a direct faecal sampling from the rectum. Another limiting factor is the naturally occurring diurnal fluctuations in egg excretion of chicken nematodes [21]. Thirdly, egg counting techniques with random faecal samples provide less reliable information for the detection and quantification of $H$. gallinarum infections [22] because this species is located in the caeca and its eggs are shed irregularly to the external environment through the caecal faeces only a couple of times per day [23, 24]. Thus, other diagnostic techniques are needed, particularly those that are host-friendly and non-invasive.

Immunity to nematode infections in chickens seems to be regulated primarily by the mechanisms involved in cell-mediated immunity $[8,25]$ as is known for mammals [26], and appears to be under strong genetic control $[4,27]$. No protection by humoral immunity was observed in birds immunized with soluble $A$. galli antigens [28]. Although the presence of circulating antibodies against a nematode species does not necessarily indicate an established protective immunity [28, 29], it indicates the presence of a past or actual infection history with nematodes. Thus, it has the potential to be used as an indication of infection and is of diagnostic importance. Serological analyses have been employed to assess the humoral immune response of chickens to A. galli infection [8, 25, 28-30]. Only Martín-Pacho et al. [30] has used an Enzyme-Linked Immunosorbent Assay (ELISA) to identify naturally infected animals; however, in that study, the sampled hens were not examined for worm burden, the current gold standard for confirming the occurrence and intensity of nematode infection, leaving the validity of the assay undetermined. Because bleeding animals to obtain plasma or serum is invasive and requires an authorized person (e.g. a veterinarian or trained technician), biological material that can be collected in a non-invasive way is both practical and important for animal welfare. In this respect, chicken eggs may be useful, as it is known that the transfer of circulating antibodies to egg yolks occurs in chickens [25, 31], analogous to cross-placental transmission in mammals [32].

Because $A$. galli and $H$. gallinarum share the same host animal, e.g. the chicken, they co-exist in most cases of naturally occurring infections $[2,3,5]$ across a wide geographic area [5] and are genetically closely related species $[6,7]$, it is, therefore, reasonable to assume that these two parasites may induce similar antigen-induced antibody responses in the chicken host. Consequently, antibodies raised against one species (e.g. A. galli) may show crossreactivity due to the existence of the other species (e.g. $H$. gallinarum) and vice versa. To test whether both parasite species induce the production of cross reactive antibodies, samples for serological analyses that are derived from both mono-species- and multi-species-infected hosts are required. We developed an ELISA based on A. galli antigens to measure antibodies raised against both A. galli and $H$. gallinarum in chicken plasma and egg yolks. We then tested two hypotheses, as follows: (i) antibodies developed against $A$. galli will, beyond cross-reactivity, also be useful to identify $H$. gallinarum-infected animals, and (ii) circulating antibodies accumulated in egg yolks are as informative as plasma samples, so that non-invasive sampling may be possible. Therefore, the aim of this study was to compare the diagnostic accuracy of the ELISA system with faecal egg counts using both plasma and egg yolk samples from experimentally infected and uninfected control birds. The validation of the assay using plasma samples from naturally multi-nematode species infected animals was additionally addressed.

\section{Methods}

Enzyme linked immunosorbent assay (ELISA)

Plasma $A$. galli-specific antibodies were determined using antigen-coated microtiter wells. Bound antibodies were 
measured by an enzyme-conjugated secondary antibody against chicken IgG (IgY). A simplified schematic representation of the ELISA format is shown in Additional file 1: Figure S1a.

\section{Antigen isolation from $A$. galli}

For antigen isolation, thawed worms were washed three times with phosphate-buffered saline (PBS), followed by one wash with $70 \%$ ethanol and another PBS wash. After homogenization in a mortar, one part of worms was extracted with two parts of a basic buffer ( $35 \mathrm{mM}$ BisTris, $25 \mathrm{mM}$ Tris, $\mathrm{pH}$ 9) for $5 \mathrm{~min}$. After centrifugation at $17000 \times g$ at $4{ }^{\circ} \mathrm{C}$ for $15 \mathrm{~min}$, the supernatant was collected (soluble antigens). The pellet was washed twice by re-suspending in the same buffer and centrifuging. This pellet was then suspended in a buffer containing $100 \mathrm{mM}$ sodium acetate, $\mathrm{pH} 5$, containing $2 \%$ SDS, $50 \mathrm{mM}$ TCEP (Fisher Scientific GmbH, Schwerte, Germany), $1 \mathrm{mM}$ EDTA and incubated at room temperature for $1 \mathrm{~h}$ before centrifugation as described above. The supernatant was collected (solubilized antigens). After protein measurement, both supernatants that contained soluble and solubilized antigens were used in a 1:1 protein ratio for coating the microtiter plates. Ascaridia galli proteins with potential antigenic properties in both soluble proteins as well as in the solubilized pellet are shown in the SDS-PAGE (sodium dodecyl sulfate polyacrylamide gel electrophoresis) analysis, provided in Additional file 1: Figure S1b.

During the initial steps of the assay development, antigens of $H$. gallinarum were also isolated as described for A. galli. Thereafter, plasma samples from birds infected either with A. galli or H. gallinarum as well as from uninfected control birds were analysed with both assay prototypes, each developed based on worm-specific antigens for the diagnosis of either A. galli or H. gallinarum (see Additional file 2: Figure S2). Because there were reasonably high correlations $\left(r_{(39-45)} \geq 0.70, P<0.0001\right)$ between plasma A. galli- and H. gallinarum-specific IgY antibody concentrations quantified with either assay, and both assays differentiated satisfactorily between infected and control birds, it was decided to use the assay developed for the diagnosis of $A$. galli because it is much easier to obtain the required antigens for the assay from the larger worm A. galli than from H. gallinarum.

\section{Assay procedures}

EIA/RIA $1 \times 8$ strip-well microtiter plates (Nunc AIS, Nunc PolySorp 469078) were coated overnight at $4{ }^{\circ} \mathrm{C}$ with $100 \mu \mathrm{l}$ A. galli extract at a concentration of $10 \mu \mathrm{g} / \mathrm{ml}$ in coating buffer $(0.05 \mathrm{M}$ sodium dihydrogen-phosphate; 0.03\% 5-Bromo-5-nitro-1.3-dioxane (BND) SDT - Stereospecific Detection Technology, Baesweiler, Germany; $\mathrm{pH}$ 7.4). After the overnight incubation, the wells were blocked with blocking buffer (20\% PBS; $0.1 \%$ tween 20;
$0.06 \% \mathrm{BND}$ ) for $1 \mathrm{~h}$ and then washed 5 times with $350 \mu \mathrm{l}$ washing buffer (1:2 diluted blocking buffer) and dried at room temperature.

Plasma samples were incubated at a dilution of 1:2,500 in assay buffer $(0.02 \mathrm{M}$ disodium hydrogen-phosphate; $0.01 \mathrm{M}$ EDTA; $0.2 \% \mathrm{BSA} ; 0.12 \mathrm{M}$ sodium chloride; $0.005 \%$ chlorhexidine di-gluconate; $0.1 \%$ gelatine hydrolysate; $0.05 \%$ Tween 20; $0.02 \%$ phenol red; 0.06\% BND) for $2 \mathrm{~h}$ on a plate shaker. After incubation, the plates were washed 5 times with washing buffer. Then, $100 \mu \mathrm{l}$ of enzyme conjugate (peroxidase conjugated goat antichicken IgG antibodies (Rockland Immunochemicals, Limerick, PA, USA) at $100 \mathrm{ng} / \mathrm{ml}$ in an assay buffer containing $10 \%$ normal goat serum (PAN-BIOTECH, Aidenbach, Germany) was incubated for $30 \mathrm{~min}$ on a plate shaker. After an additional 5 washes, the TMB substrate (TMBS, SurModics, MN, USA) was added for $30 \mathrm{~min}$ on the plate shaker, followed by termination with $100 \mu \mathrm{l}$ of $1 \mathrm{M}$ hydrochloric acid and an OD measurement at $450 \mathrm{~nm}$. Antibody binding was expressed relative to a standard chicken serum with high antibody activity $(1,000 \mathrm{mU} / \mathrm{ml}$ per definition). All further sample antibody binding results were expressed in relation to the standard chicken serum, which was used serially diluted in every assay as standard curve using a 4-parameter logistic (4-PL) for calculation of unknown samples.

The inter-assay coefficient of variability (CV) was $11.7 \%$ (4 samples, 10 assays). The overall intra-assay CV (24 samples, 2 assays) was $4.7 \%$ (3.6 and $5.7 \%$ for the first and second assays, respectively).

\section{Experimental infections}

Most of the data presented in this study originated from two independent mono-species infection experiments using the chicken host. Each experiment was performed separately with a nematode species, either A. galli or $H$. gallinarum. Plasma, egg yolk and faecal samples were collected from 106 white leghorn (Lohmann Selected Leghorn) chickens that survived until the end of the studies and were necropsied for worm burden from the two independent experiments. The birds had been purchased from a commercial hatchery as one-day-old birds and reared under helminth-free conditions until the experimental infections. The birds were kept under floorhusbandry conditions on wood-shavings as litter and fed ad libitum commercial diets to supply age-specific nutritional needs. Age of the birds at the infection was 16 and 4 weeks in A. galli and $H$. gallinarum experiments, respectively. Necropsies were then performed 28 or 30 weeks (wk) post-infection (p.i.) at an age of 44 or 34 weeks in the experiments with $A$. galli and $H$. gallinarum, respectively. In each experiment, the birds were kept either as uninfected controls or experimentally infected with 1,000 embryonated/infective eggs of either 
nematode. Numbers of birds in the uninfected control groups were 9 and 25 in A. galli and H. gallinarum experiments, respectively. Numbers of birds in the corresponding infected groups were 31 and 41, respectively.

The birds were necropsied after electrical stunning for post-mortem parasitological examinations. The small intestines and caeca were opened, and the contents were sieved through a $100 \mu \mathrm{m}$ mesh with tap water. The residues on the sieve were transferred into Petri dishes for counting larvae and adult worms of both sexes using a stereomicroscope. Experimental infection procedures and the post-mortem parasitological examinations followed the recommendations for the experimental infection of chickens [33] and were similar to previous studies with either nematode species $[11,13,34]$. Worms were classified as larvae, male and female adult worms based on general morphology. For H. gallinarum, female worms were further classified as egg-containing mature females or immatures without eggs in their uteri. Average worm length was estimated by measuring randomly selected 10 male and 10 female worms (only matures for $H$. gallinarum) of each species. Larvae were not measured. The infection experiment with $H$. gallinarum excluded Histomonas meleagridis, a (hyper-) parasite that is vectored by $H$. gallinarum and is able to parasitize both $H$. gallinarum and the definitive host e.g. the chicken [12, 16]. This was achieved by using infective $H$. gallinarum eggs obtained from worms residing in birds that were treated against $H$. meleagridis starting from 2 days before and for 7 days after the oral inoculation with infective eggs of $H$. gallinarum. The details of the methodology used for the treatment with dimetridazole against $H$. meleagridis are given in a previous report [12].

\section{Faecal, egg yolk and plasma samples}

During the final days of life, the birds were kept in individual cages for $24 \mathrm{~h}$ to collect daily total faeces and individual chicken eggs. The egg yolks were separated and stored at $-20{ }^{\circ} \mathrm{C}$. Starting from the infection day on, individual blood samples were collected fortnightly, e.g. every second week p.i. to monitor seroconversion. Blood was taken from the wing vein (vena cutanea ulnaris) into vials containing potassium-EDTA (Sarstedt AG \& Co, Nümbrecht, Germany). Blood was also collected at necropsy immediately after neck bleeding following the electrical stunning. The blood samples were centrifuged at $1619 \times g$ for $10 \mathrm{~min}$, and the plasma was stored at $-20{ }^{\circ} \mathrm{C}$ until analysis.

All the fresh daily total faeces were mixed thoroughly for each individual, and a random sub-sample $(4 \mathrm{~g})$ was analysed with a modified McMaster egg counting technique [35] to determine egg concentration in the faeces (number of eggs per gram faeces, EPG). The minimum detection level (MDL) of the technique was 50 EPG.
Further details of sample processing are described in previous studies dedicated to the quantification of egg excretion and fecundity of $H$. gallinarum [22, 34].

\section{Validation of the ELISA with plasma samples from the field}

An additional data set was derived from a field study as described below. For validation of the ELISA with field data, we analysed plasma samples obtained from birds with naturally occurring mixed-nematode infections and compared with the FECs. The plasma samples were obtained from naturally infected hens that were used in a previous study exploring genetic resistance to nematodes [4]. The vast majority (> 99\%) of the hens were infected with at least one of three most common nematodes $(A$. galli, $H$. gallinarum and Capillaria spp.). For the present study 40 hens with both faeces and plasma samples available were used. The hens originated from two genotypes of Lohmann Brown (LB; i.e. LB Classic and LB Plus) and shared highly similar genetic backgrounds, with LB Plus hens being additionally improved for a higher body size and feed intake. The faecal and plasma samples were collected from these hens at necropsy for quantification of worm burden at 79 or 88 weeks of age for the LB Classic and LB Plus genotypes, respectively. Faecal and plasma samples were examined for EPG, and the $A$. galli antibodies were measured as described for the experimentally infected birds. The hens of both genotypes were kept together on an organic farm and housed separately in two different mobile stalls. For further details of the animals, samples and infections see Wongrak et al. [3, 4].

\section{Statistics}

Worm burden, FEC, and plasma and egg yolk antibody concentrations were transformed using a natural logarithmic $(\ln )$ function $[\operatorname{Ln}(y)=\ln (y+1)]$ to correct for heterogeneity of variance and to produce approximately normally distributed data. Statistical analyses were performed using the log-transformed data separately for the A. galli and $H$. gallinarum experiments, except for the seroconversion data (see below).

Differences in plasma and egg yolk antibody concentrations between infection groups (control vs experimentally infected) were analysed with one-way ANOVA using procedure GLM of SAS [36]. Control birds were excluded from the analyses for worm burden and faecal egg count data. Correlations among worm burden and faecal egg counts as well as plasma and egg yolk antibody concentrations were analysed by Pearson correlation analysis using SAS [36]. Correlations were also based on log-transformed data.

Data consisting of plasma and egg yolk antibody concentrations as well as FEC of experimentally infected 
birds and their fully-matched uninfected controls at necropsy were used to assess diagnostic accuracy of the assay. For this purpose a Receiver Operating Characteristic (ROC) analysis was performed to determine objective non-arbitrary cut-off values, sensitivity, specificity and area-under-the-ROC-curve (AUC) as a measure of overall test accuracy [37]. The ROC analyses with a pretest probability of 50 and cost ratio of 1 as well as pairwise comparisons among ROC curve areas for each test (ELISA-plasma, ELISA-egg yolk and FEC) were performed using SigmaPlot 11.0 [38]. Because three different sample types (plasma, egg yolk and faeces) were obtained from the same individuals, pairwise comparisons of the ROC areas were performed using the method of DeLong, DeLong and Clarke-Pearson by selection of the paired data type option of SigmaPlot 11.0 [38]. Interpretations of AUC were based on the accuracy categories $[37,39]$. Briefly, accuracy of an assay is classified based on AUC either as low $(0.5<\mathrm{AUC} \leq 0.7)$ or moderate $(0.7<$ AUC $\leq 0.9)$ or high $($ AUC $>0.90)$.

An additional set of data containing information on seroconversion time (weeks required until cut-off values are achieved for the first time) in experimentally infected chickens in two studies were also analysed with oneway-ANOVA using SAS [36]. The data set constituted single time records of each experimentally infected animal recording the first time that an individual plasma antibody concentration was above the cut-off value determined for each nematode species. The records were based on an every-second-week time interval starting from the time of infection, implying that the individual seroconversion point could be detected at any post-infection week with an even number (e.g. at $2^{\text {nd }}$ or $4^{\text {th }} \ldots$ week) during the study period.

Log-transformed data obtained from the field study were analysed with one-way-ANOVA to compare plasma antibody concentrations, FECs as well as worm counts of two genotypes. By using a cut-off determined for experimentally A. galli-infected animals, true positive and false negative cases in each genotype were determined and presented graphically.

Graphical representation of all the data was performed using SigmaPlot 11.0 [38] and JMP 12 [36] software.

\section{Results}

\section{Presence and intensity of infections}

Except for one bird that was experimentally infected with A. galli, all of the infected birds harboured worms at the end of the study. This single bird was infectionpositive by FECs at an earlier time point (data not shown). On average, an A. galli-infected bird harboured $50 \pm 5.7$ (Mean \pm SE) worms, of which $22 \%$ were larvae (Table 1). Average female worm length was $93 \mathrm{~mm}$ and the proportion of female worms to male worms was in
Table 1 Worm counts and further parameters (Mean \pm SE) describing infections of chickens with Ascaridia galli or Heterakis gallinarum

\begin{tabular}{lll}
\hline Item & \multicolumn{2}{l}{ Experimental infections } \\
\cline { 2 - 3 } & A. gallib & H. gallinarum \\
\hline Host $(n)$ & 31 & 41 \\
Larva ( /bird) & $11 \pm 2.8$ & $83 \pm 11.3$ \\
Male ( $n$ /bird) & $14 \pm 1.9$ & $193 \pm 15.4$ \\
Female total ( $n$ /bird) & $25 \pm 3.4$ & $260 \pm 20.6$ \\
Immature female ( $n /$ bird) & $n d$ & $20 \pm 3.3$ \\
Mature female ( $n /$ bird) & $n d$ & $240 \pm 18.6$ \\
Total worm burden ( $n /$ bird) & $50 \pm 5.7$ & $536 \pm 42.5$ \\
Female worm length (mm) & $93 \pm 1.4$ & $9.5 \pm 0.09$ \\
Daily faeces (g/bird) & $146 \pm 6.6$ & $138 \pm 4.6$ \\
EPG ( $n / g$ faeces) & $1,803 \pm 598$ & $335 \pm 63.3$ \\
EPD ( $n$ /day) & $223,762 \pm 69,081$ & $46,777 \pm 8,821$ \\
Fecundity (EPD/female, total) & $8,928 \pm 2830$ & $218 \pm 37$ \\
Fecundity (EPD/mature female) & nd & $234 \pm 40$ \\
\hline
\end{tabular}

${ }^{a}$ Only birds that survived to the end of the experiments were included in this study

${ }^{\mathrm{b}}$ Birds were infected with 1,000 infective eggs of $A$. galli and necropsied 28 weeks p.i. at an age of 44 weeks

'Birds were infected with 1,000 infective eggs of $H$. gallinarum and necropsied 30 week p.i. at an age of 34 weeks

Abbreviations: $n d$ not determined, SE standard error

favour of females. On average, a female A. galli shed approximately 9,000 eggs within $24 \mathrm{~h}$ through $146 \mathrm{~g}$ faeces (Table 1).

Overall, the average $H$. gallinarum burden was $536 \pm 42.5$ worms per bird, and the larval stages accounted for $15 \%$ of the worm population (Table 1). Immature female worms constituted approximately 8 and $4 \%$ of all females and all worms, respectively. Proportion of female (mature + immature) to male worms was in favour of females. Faecal egg concentration was on average $335 \pm 63.3$ EPG and each animal excreted daily approximately $138 \mathrm{~g}$ faeces, which then represented a fecundity estimate of 234 eggs per mature female worm within $24 \mathrm{~h}$.

\section{Plasma and egg-yolk IgY antibody}

The assay quantified large differences in plasma or in egg yolk IgY levels between infected and uninfected animals in two experiments, each performed with either of the nematode species. Overall average plasma and eggyolk antibody concentrations of the uninfected and infected birds in each experiment are summarized in Additional file 3: Table S1. As shown in Fig. 1a, there was a significant effect of $A$. galli infection on $A$. galli-specific antibody concentrations in plasma samples $\left(F_{(1,39)}=38.34\right.$, $P<0.0001)$. Similarly, $H$. gallinarum infected birds had higher $\left(F_{(1,65)}=50.30, P<0.0001\right)$ antibody levels as compared to their age-matched uninfected controls. Similar to 


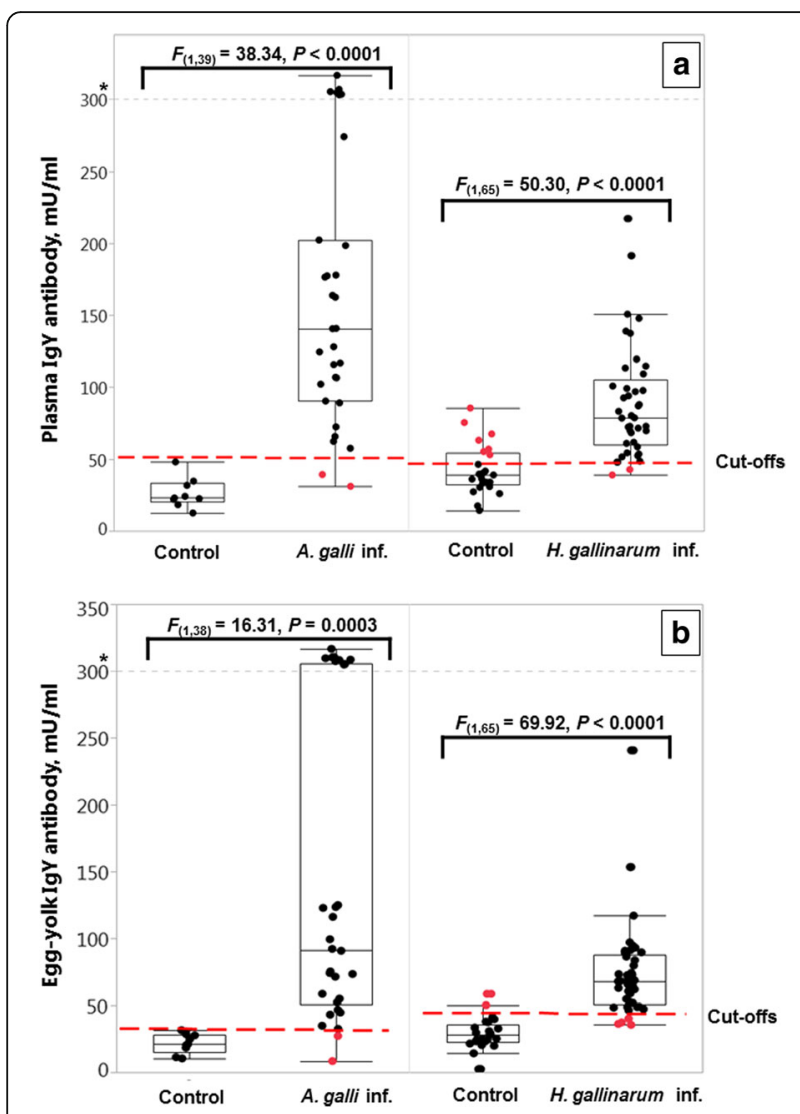

Fig. 1 Plasma (a) and egg-yolk (b) lgY concentrations in uninfected or experimentally infected birds in two experiments. Infected birds received an oral inoculation of 1,000 embryonated eggs of $A$. galli or H. gallinarum and necropsied 28 or 30 week p.i., respectively. Data are shown with the outlier box plots with the vertical line within the box representing the sample median, and the lower and upper end of the box representing the $25^{\text {th }}$ and $75^{\text {th }}$ quantiles, respectively. Each dot is an independent observation. The extent of the whiskers is calculated as $25^{\text {th }}$ and $75^{\text {th }}$ quartiles plus or minus $1.5 \times$ interquartile range, respectively. Outliers above the grey line $(*>300)$ are shown on an extended reduced scale [reduced $y$-axis $=\left(y^{*} 0.01+300\right)$ ] to depict a focused picture of the whole data. The dashed red lines indicate the cut-off values quantified by the ROC analyses for each nematode and sample matrix (see Table 2 for details). The figure represents raw data, but the statistical comparisons are based on the log-transformed data

plasma samples, both A. galli $\left(F_{(1,38)}=16.31, P=0.0003\right)$ and $H$. gallinarum $\left(F_{(1,65)}=69.92, P<0.0001\right)$ monoinfections resulted in an elevated level of antibody concentration in the egg-yolks of the infected birds (Fig. 1b).

\section{Correlations between worm burden and infection proxies}

The total worm burden did not significantly correlate with plasma or egg yolk antibody concentrations of the chickens infected with either of the nematodes $(P>0.05)$. There was a moderate-high positive correlation $\left(r_{(31)}=0.42\right.$, $P=0.0191$ ) between EPG and total worm burden in experimentally $A$. galli-infected chickens, whereas the correlation between EPG and worm burden for $H$. gallinarum was not significantly different from zero $\left(r_{(41)}=-0.14, P=0.3847\right)$.

\section{ROC curve analyses}

The area under the ROC curve (AUC $\pm \mathrm{SE}$ ), a measure of total test accuracy, indicated that the assay performed with high accuracy (AUC > 0.90) for both nematodes using either plasma or egg yolks (Fig. 2 and Table 2). Total test accuracy of the assay with plasma samples (AUC $=0.99 \pm 0.014)$ tended to be higher $\left(\chi^{2}=3.457, d f=\right.$ $1, P=0.0630)$ than FEC (AUC $=0.92 \pm 0.033$ ) for $A$. galli (Table 2). Sensitivity of the assay was 94 and $93 \%$ with plasma and egg yolk samples, respectively, whereas FEC yielded in a sensitivity of $84 \%$ in A. galli experiment. Both the ELISA and FEC identified true negative animals with the highest possible specificity (100\%) in the A. galli experiment.

Although the assay tended $\left(\chi^{2}=2.921, d f=1, P=0.0874\right)$ to perform better with egg-yolks $(\mathrm{AUC}=0.96 \pm 0.019)$ than with plasma ( $\mathrm{AUC}=0.91 \pm 0.037$ ) in $H$. gallinarum experiment, no significant difference was quantified when the assay with either sample type was compared with FEC (AUC $=0.95 \pm 0.023$ ). Sensitivity of the assay was 95 and 90\% with plasma and egg yolk samples, respectively whereas FEC derived from $24 \mathrm{~h}$ faeces samples provided a sensitivity of $90 \%$ (CI: 77-97\%). None of the negative faeces samples was found to be positive with FEC, whereas ELISA classified falsely 8 and $28 \%$ of uninfected controls as H. gallinarum-infected by using egg-yolks and plasma samples, respectively (Fig. 1 and Table 2).

\section{Seroconversion}

As shown in Fig. 3, the time required to quantify a sufficient level of antibodies for the first time (seroconversion) in plasma samples with the species-specific cut-off values (see Table 2 ) was significantly $\left(F_{(1,71)}=44.28, P<0.0001\right)$ lower for A. galli infected animals (5.8 \pm 0.77 weeks) than for $H$. gallinarum infected animals $(10.9 \pm 0.32$ weeks). Plasma antibody levels of more than $80 \%$ of $A$. galli-infected birds exceeded the cut-off values already by the $4^{\text {th }}$ week of infection. $H$. gallinarum-infected birds showed seroconversion for the first time by the $8^{\text {th }}$ week of infection. All the birds infected with either nematode were positively (> cut-off) identified latest by 18 weeks p.i. (Fig. 3).

\section{Validation of the ELISA with naturally occurring mixed infections}

All the naturally infected hens harboured both $H$. gallinarum and $A$. galli, whereas $86 \%$ of the birds were also infected with Capillaria spp. (Table 3). On average (Mean \pm SE) each hen harboured $240 \pm 41.8$ worms with a high variation ranging from 17 to 1,451 worms/hen. Percentages of true positive animals using the cut-off value $(52.9 \mathrm{mU} / \mathrm{ml})$ that was determined by the ROC 

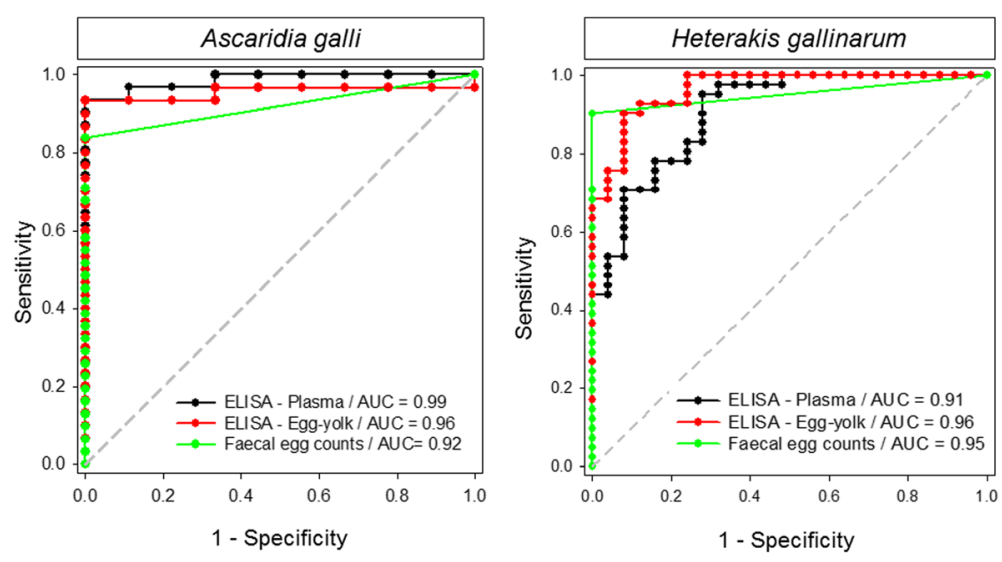

Fig. 2 Overall diagnostic accuracy of the ELISA using plasma or egg-yolk samples in comparison to FECs. Area under the ROC curves (AUC) for the ELISA assays based on plasma antibody, egg yolk antibody or for faecal egg counts in chickens experimentally infected with A. galli or H. gallinarum. The dashed diagonal line corresponds to the half of the maximum AUC (1.0). The farther the location of the ROC curve is from the diagonal line, the higher the total test accuracy

analyses for experimentally $A$. galli infected animals were $90 \%$ across two genotypes (Fig. 4). Out of 40 naturally infected hens from which both faeces and plasma samples were obtained, 18 (45\%) were negative with FEC (Fig. 4). Overall average plasma antibody concentration of the naturally infected hens was $120 \pm 9.1$ (Table 3). Although the plasma antibody concentration was higher $\left(F_{(1,39)}=6.77, P=0.013\right.$; Fig. $\left.5 \mathrm{a}\right)$ in LB Plus than in LB Classic hens, there was no significant difference $\left(F_{(1,39)}=\right.$ $0.17, P=0.687$; Fig. $5 b$ ) in faecal egg concentrations (EPG) between the two genotypes. Average EPG in the naturally infected hens across the two genotypes was $279 \pm 60$ (Table 3). Total worm burdens of the two genotypes did not differ significantly $\left(F_{(1,39)}=0.08, P=0.774\right)$.

\section{Discussion}

The two hypotheses examined in the present study could not be rejected. It was shown that the ELISA based on somatic antigens of $A$. galli not only identified with high accuracy animals infected with this nematode species but also those infected with $H$. gallinarum, indicating the production of cross-reacting antibodies in chickens against both worm species. The ELISA provided a higher diagnostic test accuracy, particularly for naturally infected animals from the field, compared with the FECs. The results also demonstrated that the IgY antibodies found in egg yolks are as informative as plasma samples; therefore, host friendly, non-invasive sampling is possible. Nevertheless, the quantitative informative ability of the assay is limited compared with FECs, which show a higher correlation with the actual A. galli infection intensity (e.g. A. galli burden) of the birds.

\section{Intensity of infections and comparability with natural ones} Despite the importance of re-emerging nematode infections in chickens, diagnostic tools for these infections are mainly limited to coprological analyses with relatively low accuracy [3] and precision [22]. Although the pig industry is facing a similar infection threat from roundworms, particularly Ascaris suum, a successful

Table 2 Diagnostic ability of the ELISA using plasma or egg yolk samples in comparison to faecal egg counts (FEC) obtained from $24 \mathrm{~h}$ faeces in chickens with mono-Ascaridia galli or -Heterakis gallinarum infections

\begin{tabular}{lllll}
\hline Worm species & Parameter & ELISA - Plasma & ELISA - Egg yolk & FEC \\
\hline A. galli & AUC \pm SE & $0.99 \pm 0.014^{\mathrm{a}}$ & $0.96 \pm 0.034$ & $0.92 \pm 0.033^{\mathrm{a}}$ \\
& Cut-off & $52.9 \mathrm{mU} / \mathrm{ml}$ & $32.0 \mathrm{mU} / \mathrm{ml}$ & $25 \mathrm{EPG}$ \\
& Sensitivity, \% $(95 \% \mathrm{Cl})$ & $94(79-99)$ & $93(78-99)$ & $84(66-95)$ \\
& Specificity, \% (95\% Cl) & $100(66-100)$ & $100(66-100)$ & $100(66-100)$ \\
H. gallinarum & AUC \pm SE & $0.91 \pm 0.037^{\mathrm{b}}$ & $0.96 \pm 0.019^{\mathrm{b}}$ & $0.95 \pm 0.023$ \\
& Cut-off & $47.2 \mathrm{mU} / \mathrm{ml}$ & $43.8 \mathrm{mU} / \mathrm{ml}$ & $25 \mathrm{EPG}$ \\
& Sensitivity, \% (95\% Cl) & $95(83-99)$ & $90(77-97)$ & $90(77-97)$ \\
& Specificity, \% (95\% Cl) & $72(51-88)$ & $92(74-99)$ & $100(86-100)$
\end{tabular}

${ }^{a}$ Area under the ROC curves (AUC) sharing the superscript tend to differ between corresponding tests in $A$. galli infection (Chi-square test: $\left.\chi^{2}=3.457, d f=1, P=0.0630\right)$

${ }^{\mathrm{b}} \mathrm{AUC}$ sharing the superscript tend to differ between corresponding tests in $\mathrm{H}$. gallinarum infection $\left(\mathrm{Chi}-\mathrm{square}\right.$ test: $\left.X^{2}=2.921, d f=1, P=0.0874\right)$ 


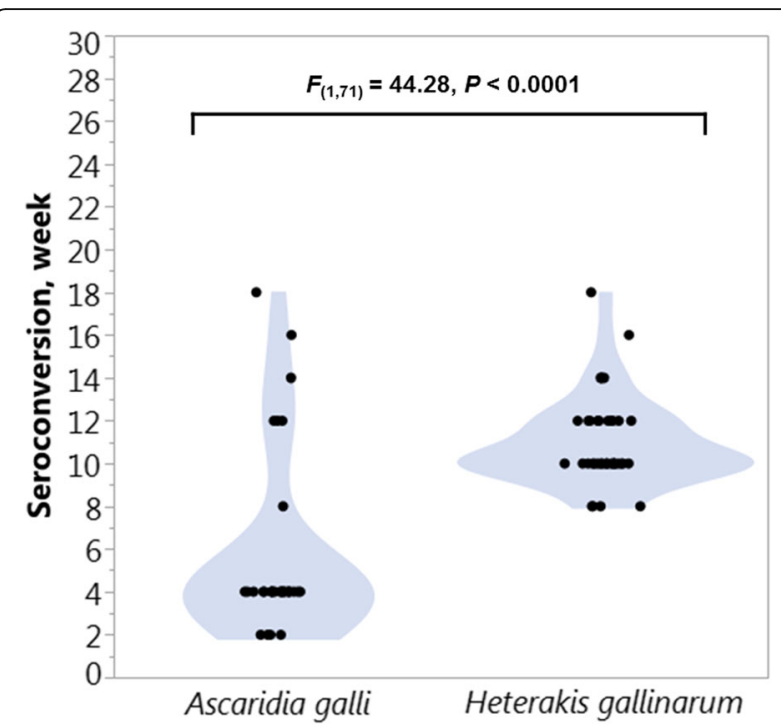

Fig. 3 Seroconversion with plasma IgY and worm specific cut-off values. Contour-plots showing seroconversion for experimentally $A$. galli- or $\mathrm{H}$. gallinarum-infected chickens using plasma samples and worm specific cut-off values for plasma samples (52.9 and $47.2 \mathrm{mU}$ / $\mathrm{ml}$ for A. galli and $\mathrm{H}$. gallinarum, respectively). Notice that the $\mathrm{Y}$-axis is scaled to the duration of the $\mathrm{H}$. gallinarum experiment ( 30 week). For the A. galli experiment, the duration was 28 weeks

assay has been recently developed to identify infected animals $[40,41]$. The data reported in the experimental part of this study were derived from animals with a long-history ( $\geq 28$ weeks) of infection. The life-cycle of H. gallinarum and A. galli can be completed as early as 5 and 8 weeks, respectively, with both nematodes requiring a similar period of time for their eggs to become fully embryonated [42, 43], although $A$. galli has a longer prepatent period [11, 34]. Both A. galli and H. gallinarum infections were patent and included re-infections, as

Table 3 Descriptive statistics for worm burdens, faecal egg counts and plasma antibody concentrations of field chickens ${ }^{a}$ naturally infected with gastrointestinal nematodes

\begin{tabular}{llrlll}
\hline Item & Mean & SE & Min & Max & Prevalence $(\%)^{b}$ \\
\hline H. gallinarum $(n /$ bird $)$ & 192 & 40.9 & 3 & 1408 & 100 \\
A. galli $(n /$ bird $)$ & 36 & 6.0 & 1 & 126 & 100 \\
Capillaria spp. (n/bird) & 13 & 2.2 & 0 & 52 & 86 \\
Total worm burden $(n /$ bird) & 240 & 41.8 & 17 & 1451 & 100 \\
EPG & 279 & 60.0 & 0 & 1278 & 55 \\
Plasma antibody $(\mathrm{mU} / \mathrm{ml})$ & 120 & 9.1 & 30.6 & 236.6 & 90
\end{tabular}

${ }^{a}$ The chickens $(n=40)$ were brown laying hens of two closely related genotypes, kept on an organic farm and necropsied in the end of laying period at an age of $\geq 79$ weeks

${ }^{\mathrm{b}}$ Percentage of chickens infected with each or any nematode species. For EPG it indicates percentage of chickens with EPG positive samples (e.g. EPG $\geq 50$ ). For plasma antibody it indicates percentage of animals correctly classified as infected using the cut-off (> $52.9 \mathrm{mU} / \mathrm{ml}$ )

'Calculated as the sum of all worms from three nematode species. For calculation of prevalence, the existence of at least one species was considered to be positive Abbreviations: Min minimum, Max maximum, SE standard error

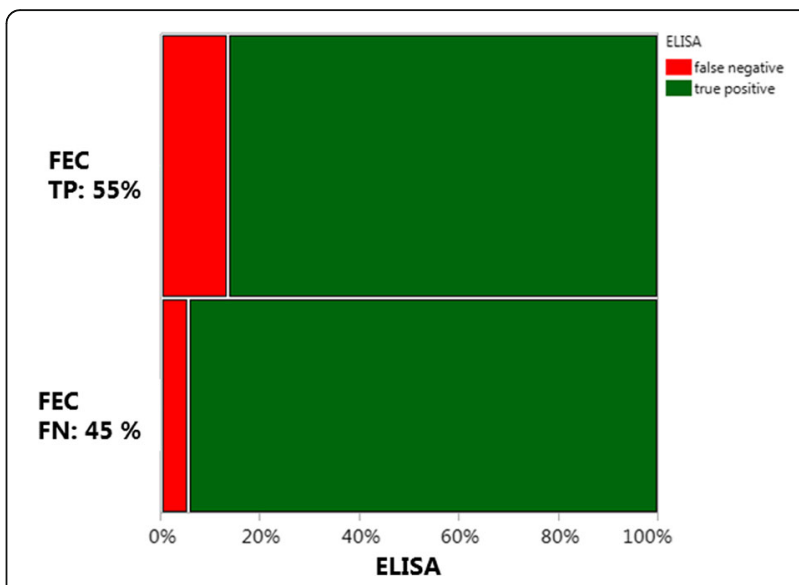

Fig. 4 Qualitative comparison of the ELISA with FECs using naturally infected animals from the field. A mosaic plot of the field data representing two-way percentages of false negative (FN) and true positive (TP) cases for naturally infected hens identified with faecal egg counts (FEC) and ELISA

indicated by the presence of larvae that accounted for approximately 15 and $22 \%$ of $H$. gallinarum and A. galli populations, respectively. Average worm burdens with either parasite were higher than reported in our previous experimental infection studies [11, 13, 34], most likely due to the longer period of time in this study, allowing reinfections to occur continuously. However, the per capita worm fecundity (number of eggs excreted per female within a day) was much lower ( 44 and $76 \%$ for $H$. gallinarum and A. galli, respectively) than previously reported for both species in chickens with lower infection intensities [21,34], indicating a strong density-dependency in worm fecundity from crowded populations. Infection intensities resulting from the mono-species inoculations were indeed even higher than the levels quantified in the field study. As re-infections must have occurred continuously, the higher worm burdens with both species not only indicate the effectiveness of the oral-faecal transmission route of the infections but probably also point to the lack of an effective acquired immunity against both nematodes. Because the intensities of infections resulting from the experimental inoculation of eggs and/or naturally occurring re-infections were even higher than the levels quantified in the field study, and the worm populations consisted of both mature and juvenile stages, we infer from the results that the experiments were highly comparable to those occurring under natural conditions, with the exception that all the birds were exposed to infections with monospecies. The mono-species infections enabled us to compare the diagnostic accuracy of the ELISA for both worm species using plasma and egg yolk samples in comparison to a commonly used faecal egg counting technique (e.g. McMaster technique). 


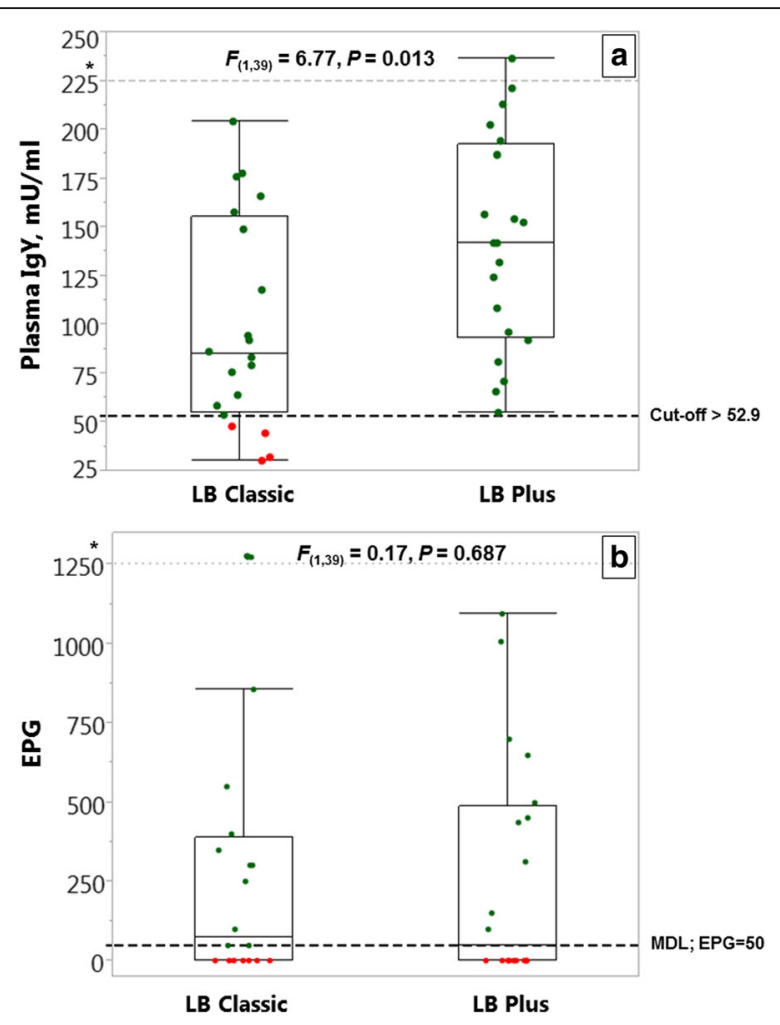

Fig. 5 Plasma antibody (a) and FECs (b) in naturally infected chickens from the field. Plasma samples $(n=40)$ were obtained from chickens with naturally occurring nematode infections that were used in a previous study exploring host genetic resistance to nematodes [4]. The dashed line, indicating the cut-off value $(52.9 \mathrm{mU} / \mathrm{ml})$, was determined by the ROC analyses for experimentally $A$. galli infected animals and used here as the threshold to classify the hens as infected

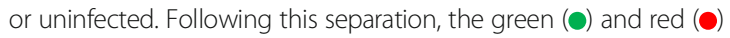
dots represent true and false positive cases, respectively. Outliers above the dashed grey line $\left(^{*}>300\right)$ are shown on an extended reduced scale [reduced $y$-axis $\left.=\left(y^{*} 0.01+300\right)\right]$ to depict a focused picture of the whole data. The FECs (faecal egg counts) are expressed as EPG (number of eggs per gram faeces). MDL: minimum detection limit of the McMaster egg counting technique $(E P G=50)$. For the properties of the box plots, see the explanations given in Fig. 1. The figure represents raw data, but the statistical comparisons are based on the log-transformed data

\section{Diagnostic ability of the ELISA}

The total diagnostic test accuracy of the ELISA, as quantified with area under curve of the ROC analyses, was high for both nematodes using plasma or egg yolks as sample matrix (AUC > 0.90). Although accuracy of ELISA with plasma samples $(\mathrm{AUC}=0.99)$ tended to be higher than that of FECs $(\mathrm{AUC}=0.92)$ in $A$. galli experiment, there was no significant difference in $H$. gallinarum experiment. It is of crucial importance to note that the faecal samples collected in the infection experiments were not randomly taken, as their informative ability would otherwise be strongly limited due to diurnal fluctuations in egg excretion of the nematodes [21] and due to faeces-related factors $[13,22]$. They were well-mixed samples that were derived from $24 \mathrm{~h}$ faeces, which high probably prevented a strong underestimation of the test accuracy by FECs, particularly for $H$. gallinarum, as was the case for naturally multi-species-infected animals from the field. Whilst $90 \%$ of the naturally infected hens were correctly identified with the ELISA, $45 \%$ of the infected hens tested (false) negative with the FEC (Fig. 4). Both the ELISA and FEC tests were able to identify true negative animals with the highest possible specificity $(100 \%)$ in the A. galli-experiment, whereas $8-28 \%$ of uninfected control birds were falsely classified with ELISA in the $H$. gallinarum experiment, altough no significant difference was quantified in total test accuracy (AUC) between ELISA and FEC. The specificity of the ELISA with plasma samples (72\%) was somewhat lower than with egg yolks (92\%) for H. gallinarum. The high specificities of FECs (100\%) are explained by the fact that nematode eggs can only be found in faeces of infected animals harbouring mature/fecund female worms, although false positive cases have been reported for uninfected pigs practicing coprophagy [44]. The diagnostic success of the ELISA in the field will not depend only on high test sensitivity and specificity to distinguish correctly between infected and non-infected animals, but also on the true prevalence of the nematode infections, which are extremely high [1-5]. This is because of the fact that the likelihood of false-positive and falsenegative testing classification errors is strongly influenced by the true prevalence of infections [45]. Consequently, an assay with high specificity and sensitivity would not be that valuable if true prevalence of an infection is low, mainly because positive predictive value (i.e. probability of infection given a positive test result) decreases as prevalence decreases [45].

Ascaridia galli-infected animals required a shorter seroconversion time (5.8 weeks) than $H$. gallinarum-infected animals (10.9 weeks). Most of $A$. galli-infected animals (> 80\%) were classified correctly as infected already by the second sampling time (e.g. 4 weeks p.i.) indicating the potential of the assay to be used for diagnosis of early-stage $A$. galli infections, which cannot be determined by faecal egg counts as it takes 5 to 8 weeks for the infection to become patent [46].

The difference in seroconversion time may at least partly result from the age of chickens used for the experimental infections. $H$. gallinarum infected birds were younger than $A$. galli-infected birds. The younger age of the birds may be associated with the lower IgY antibody production, as is known for chickens [47]. Ascaridia galli is known to have a mucosal/histotrophic phase up to 54 days [48], which is considered to be a normal part of its life-cycle [49]. In contrast, $H$. gallinarum has a very short tissue-associated phase maximum up to 12 days [50]. As the tissue-embedded larvae may induce a higher antigenic stimulation [25], the shorter 
seroconversion time for A. galli may also partly be due to presence and duration of the mucosal/histotrophic phase. The seroconversion time might have been influenced not only by the mucosal/histotrophic phase and age of animals but also by level of infections as well as by the number of continuously occurring exposures or re-infections.

\section{One assay for diagnosis of two nematodes}

The two prototype assays, each developed for diagnosis of either A. galli or H. gallinarum, showed good agreement (see Additional file 2: Figure S2). The presence of antibodies that could be detected by either assay was a strong indication that chickens develop similar, if not the same, antibodies that cross-react to both nematode species. The molecular weight of antigens involved in the reactivity against both embryonated egg antigens and against adult $A$. galli antigens have been described by Marcos-Atxutegi et al. [25]. According to these authors, A. galli antigens responsible for the stimulation of specific IgY are polypeptide molecules of 30-34, 44-55 and $58-90 \mathrm{kDa}$. These results are in agreement with our data for A. galli somatic antigens (Additional file 1: Figure S1b) and are supported also by the data of [51] who observed antigenic bands with molecular weights of 55-72 kDa. By using a solubilisation protocol, we were able to increase the number of potential antigens, especially in the range of $40-220 \mathrm{kDa}$, to increase the spectrum of measurable worm antibodies, resulting in fewer false negative results. Although the chemical structures of these antigens are not fully known, Jordanova et al. [52] described a protein (Ag-Ibp55) with a molecular weight of $55 \mathrm{kDa}$ that represents a novel type of lipid-binding protein and may act as the nematode antigen. Although similar data are not available for $H$. gallinarum, our results indicate that the chicken host does not differentiate between somatic antigens of both nematodes, and produces similar, if not the same, antibodies that can be quantified with the same assay. This implies the non-specificity of the IgY antibodies measured by ELISA for either worm species. Despite the longer seroconversion for $\mathrm{H}$. gallinarum than for A. galli, the assay can be used generally to identify animals infected with either ascarid, which are involved in naturally occurring infections, mostly together $[2,3,5]$. The similarity in antigenic stimulations induced by $A$. galli and $H$. gallinarum may be related to the phylogenetic closeness of the two species $[6,7]$.

\section{Measuring antibodies in egg yolk}

IgY is the avian equivalent of mammalian IgG [53]. IgG and IgY are functionally homologous while having fundamental structural differences in their molecules [31, 54]. The transfer of IgY from hen serum via the yolk to the circulation of the embryo is also analogous to cross-placental transmission in mammals with IgG [32]. Although IgA and IgM are mainly found in the albumen, IgY is stored in the egg yolk [32, 54]. Our recent data indicate a very strong correlation $(r=0.89)$ between plasma and egg yolk concentrations of IgY in A. galli infected chickens [55]. The objectively determined cut-off values were smaller for egg yolk samples than for plasma samples in both A. galli and $H$. gallinarum experiments, indicating smaller minimum detection levels for the egg yolk samples. One of the features of IgY is that their half-life ranges from 36 to $65 \mathrm{~h}$ [56], which is substantially shorter than that of IgG in mammalians [54, 57], e.g. 15 days in sheep [58]. The shorter half-life of circulating IgY, which may cause fluctuations in plasma concentrations over time [56], may explain why egg yolks provided a higher test accuracy than the plasma samples for $H$. gallinarum. From an animal welfare point of view, both captivity for the collection of individual faecal samples and puncturing birds for the collection of blood to be used for serological analyses are not favourable. As the egg yolk IgY antibodies provided high test accuracy with both nematodes $(\mathrm{AUC}=0.96)$, and the eggs can be collected from the animals in a non-invasive, host-friendly way, we suggest the ELISA system with egg yolk samples as an accurate diagnostic test for identifying A. galli and H. gallinarum infected chickens. Because the egg samples were taken at the end of the study period, the transfer time of IgY to egg yolks remains to be clarified in further studies.

\section{Quantitative infection proxies}

Although there were multiple-fold and statistically significant differences in the quantity of antibody levels (plasma and egg yolk) between infected and uninfected animals (depending on parameter and worm species, on average 2 to 14 times higher), the ELISA system has not yet fully been proven to function quantitatively. For this, studies including sentinel birds necropsied at regular intervals to monitor changes in the relationship between worm burden with changing demographic composition (e.g. larval stages and first generation mature worms resulting from experimental infections and the mixed stage-populations due to re-infections) and antibody levels are required.

Among the three tests, FECs correlated better with worm burdens than the ELISA with plasma or egg yolks for both nematodes in A. galli experiment. In H. gallinarum experiment, however, there was no significant correlation between worm burden and FEC, indicating inadequacy of FECs to quantify infection intensity for this nematode even if based on well-mixed $24 \mathrm{~h}$ faecal samples. Although the FECs provided relatively high qualitative-diagnostic information (sensitivity 90\%), no reliable quantitative information on the infection intensity of $H$. gallinarum could be derived by FECs. This might at least partly be related to the impaired fecundity of female worms in crowded worm 
populations as discussed earlier. Because it is known that both inverse- and density dependency mechanisms regulate fecundity of $H$. gallinarum [34], the expected linear relationship between worm burden and FECs might have simply been masked as a consequence of the existence of different infra-populations under influence of either inverse-density dependency or density dependency effects in the entire worm population.

The results reported for correlation analysis included data only from experimentally mono-species infected animals. To mimic infections under natural conditions, where infected and uninfected animals are present in the same population, we additionally pooled data from two stratified groups (i.e. infected and uninfected animals) for correlation analysis to check whether the infection proxies (i.e. plasma and egg yolk antibody and EPG) would correlate with worm burden. This is somewhat against the statistical rationale behind data pooling but fits the natural conditions. In this way, we estimated positive correlations between A. galli counts and antibody concentrations for plasma $\left(r_{(40)}=0.65, P<0.0001\right)$ and egg yolks $\left(r_{(39)}=0.60, P<0.0001\right)$, respectively (Additional file 4: Figure S3). The corresponding correlations for $H$. gallinarum were highly positive $\left(r_{(66)}=\right.$ $0.67, P<0.0001$ and $r_{(66)}=0.73, P<0.0001$, respectively). Following this scenario, correlations between EPG and worm burden also improved for both nematodes, i.e. $r_{(40)}=0.73(P<0.0001)$ and $r_{(66)}=0.83(P<0.0001)$ for $A$. galli and $H$. gallinarum, respectively. However, as the infection intensity estimates should only apply to infected animals (e.g. animals with worm burden $\geq 1$ ), the correlations including uninfected control birds are, probably, of limited practical use.

\section{Conclusion}

We conclude that antibodies developed against $A$. galli can successfully be used to identify both A. galli- and $H$. gallinarum-infected animals with the newly developed ELISA system. Antibodies accumulated in egg yolks are as informative as plasma samples; therefore, a host friendly, non-invasive sampling is possible. Although the assay with plasma samples reveals qualitative information of higher quality than the faecal egg counts on the infection status of naturally infected birds, the latter is still a better tool to assess the intensity of $A$. galli but not of $H$. gallinarum infections in chickens.

\section{Additional files}

Additional file 1: Figure S1. a The ELISA system based on enzymeconjugated secondary antibody against chicken $\lg G(\lg Y)$ bound to micro-wells coated with the A. galli antigen. $\mathbf{b}$ Analysis of $A$. galli proteins with potential antigenic properties in both soluble proteins and in solubilized pellet extracts by electrophoresis (SDS-PAGE analysis). For the ELISA, both fractions were pooled to increase antibody-detectable antigens. (TIF $136 \mathrm{~kb}$ )

Additional file 2: Figure S2. Plasma antibody concentrations ( $\mathrm{mU} / \mathrm{ml}$ ) in chickens experimentally infected (red dot) with A. galli (A) or $\mathrm{H}$. gallinarum (B) or from uninfected control birds (green dot). Plasma samples were analysed both with the worm-specific ELISA and with the alternative assay developed for the detection of antibodies against the other worm species (A. galli vs H. gallinarum or vice versa). Antibody concentrations measured with the species-specific assay are shown on the $Y$-axes, whereas results with the alternative assay are shown on the $X$-axes of the corresponding plots. The fitted lines (dark blue line) are shown together with the confidence regions. Number of observations: a Control $(n=8)$; Infected $(n=37) ; \mathbf{b}$ Control $(n=19) ;$ Infected $(n=20)$. The figure represents raw data, but the statistical comparisons are based on the log-transformed data. (TIF $111 \mathrm{~kb}$ )

Additional file 3: Table S1. Average (Mean \pm SE) plasma and egg-yolk antibody concentrations of infected birds and their uninfected-control counterparts in A. galli and H. gallinarum experiments. (DOC $29 \mathrm{~kb}$ )

Additional file 4: Figure S3. Relationships between worm burdens and infection proxies based on pooled data. Linear relationships between worm burdens with plasma antibody, egg yolk antibody and faecal egg counts in chickens experimentally infected (red dot) with Ascaridia galli or with Heterakis gallinarum and uninfected control birds (green dot). Note that the correlations are based on pooled data from infected and uninfected controls within each nematode infection. (TIF $161 \mathrm{~kb}$ )

\section{Abbreviations}

AUC: area under curve; ELISA: Enzyme-Linked Immunosorbent Assay; EPG: number of eggs per gram of faeces; FEC: faecal egg counts; MDL: minimum detection level; ROC: Receiver Operating Characteristic

\section{Acknowledgements}

We thank Mr. Erwin Tönges and Mrs. Adelaide Teixeira for their invaluable assistance in conducting this study.

\section{Funding}

This study was funded by a grant obtained from the German "Bundesministerium für Wirtschaft und Technologie" through the funding programme 'Zentrales Innovationsprogramm Mittelstand - ZIM' with the project number KF2484202. The publication of this article was funded by the Open Access fund of the Leibniz Institute for Farm Animal Biology (FBN).

\section{Availability of data and materials}

Raw data from this study have not been shared at this time because we anticipate additional publications exploring some of the results obtained from the two independent infection experiments.

\section{Authors' contributions}

GD and MG conceived the study. MH developed the ELISA systems. GD, BS, $S R$, MS and MG induced the experimental infections and performed the experiments with chickens. Samples from the experimental studies were analysed by GD, SR and MS. KW and BS collected plasma samples from the field study, SR analysed these samples. KW, BS and GD determined worm burdens and FECs of the field animals. GD conducted the statistical analysis of all the data. GD and MH interpreted the data. GD drafted the manuscript. $M G$ and $M H$ reviewed the draft manuscript. MG and $\mathrm{MH}$ obtained funding. All authors read and approved the final manuscript.

\section{Competing interests}

All authors declare that they have no competing interests. $\mathrm{MH}$ is working in a medical company network (TECOmedical Group) that develops and sells serological tests, without any competing interests.

\section{Consent for publication}

Not applicable.

Ethics approval and consent to participate

All the experimental procedures and animal handling followed the animal welfare rules. The results presented in this study are part of a large data set 
obtained from two independent experiments performed within a project approved by the relevant ethics committee authority for animal experimentation (Lower Saxony State Office for Consumer Protection and Food Safety, Germany; Permission no: AZ:33.9-42502-04-12/0707).

\section{Publisher's Note}

Springer Nature remains neutral with regard to jurisdictional claims in published maps and institutional affiliations.

\section{Author details \\ ${ }^{1}$ Institute of Nutritional Physiology 'Oskar Kellner', Leibniz Institute for Farm Animal Biology, Wilhelm-Stahl-Allee 2, 18196 Dummerstorf, Germany. ${ }^{2}$ TECOdevelopment GmbH, Marie-Curie-Str. 1, 53359 Rheinbach, Germany. ${ }^{3}$ Department of Animal Sciences, University of Göttingen, Albrecht-Thaer-Weg 3, 37075 Göttingen, Germany. ${ }^{4}$ Faculty of Agriculture and Life Science, Chandrakasem Rajabhat University, 39/1 Ratchadaphisek Road, Chatuchak, 10900 Bangkok, Thailand. ${ }^{5}$ Free University of Bozen - Bolzano, Faculty of Science and Technology, Universitätsplatz 5, 39100 Bolzano, Italy.}

Received: 29 September 2016 Accepted: 29 March 2017

Published online: 18 April 2017

\section{References}

1. Permin A, Bisgaard M, Frandsen F, Pearman M, Nansen P, Kold J. Prevalence of gastrointestinal helminths in different poultry production systems. $\mathrm{Br}$ Poult Sci. 1999:40:439-43.

2. Kaufmann F, Daş G, Sohnrey B, Gauly M. Helminth infections in laying hens kept in organic free range systems in Germany. Livest Sci. 2011;141:182-7.

3. Wongrak K, Daş G, Moors E, Sohnrey B, Gauly M. Establishment of gastrointestinal helminth infections in free-range chickens: a longitudinal on farm study. Berl Munch Tierarztl Wochenschr. 2014;127(7-8):10-7.

4. Wongrak K, Daş G, von Borstel-König U, Gauly M. Genetic variation for worm burdens in laying hens naturally infected with gastrointestinal nematodes. Br Poult Sci. 2015;56(1):15-21.

5. Thapa S, Hinrichsen LK, Brenninkmeyer C, Gunnarsson S, Heerkens JL, Verwer $C$, et al. Prevalence and magnitude of helminth infections in organic laying hens (Gallus gallus domesticus) across Europe. Vet Parasitol. 2015; 214(1-2):118-24

6. Nadler SA, Carreno RA, Mejía-Madrid H, Ullberg J, Pagan C, Houston R, Hugot JP. Molecular phylogeny of clade III nematodes reveals multiple origins of tissue parasitism. Parasitology. 2007;134:1421-42.

7. Wang BJ, Gu XB, Yang GY, Wang T, Lai WM, Zhong ZJ, Liu GH. Mitochondrial genomes of Heterakis gallinae and Heterakis beramporia support that they belong to the infraorder Ascaridomorpha. Infect Genet Evol. 2016;40:228-35

8. Schwarz A, Gauly M, Abel H, Daş G, Humburg J, Rohn K, et al. Immunopathogenesis of Ascaridia galli infection in layer chicken. Dev Comp Immunol. 2011;35:774-84.

9. Schwarz A, Gauly M, Abel H, Daş G, Humburg J, Weiss A, et al. Pathobiology of Heterakis gallinarum mono- and co-infection with Histomonas meleagridis in layer chickens. Avian Pathol. 2011:40:277-87.

10. Luna-Olivares LA, Kyvsgaard NC, Ferdushy T, Nejsum P, Thamsborg SM, Roepstorff A, Iburg TM. The jejunal cellular responses in chickens infected with a single dose of Ascaridia galli eggs. Parasitol Res. 2015;114(7):2507-15.

11. Daş G, Kaufmann F, Abel H, Gauly M. Effect of extra dietary lysine in Ascaridia galli-infected grower layers. Vet Parasitol. 2010;170:238-43.

12. Daş G, Abel H, Humburg J, Schwarz A, Rautenschlein S, Breves G, Gauly M. Non-starch polysaccharides alter interactions between Heterakis gallinarum and Histomonas meleagridis. Vet Parasitol. 2011;176:208-16.

13. Daş G, Abel H, Rautenschlein S, Humburg J, Schwarz A, Breves G, Gauly M. The effects of dietary non-starch polysaccharides on Ascaridia galli infection in grower layers. Parasitology. 2012;139:110-9.

14. Ramadan HH, Abou Znada NY. Morphology and life history of Ascaridia galli in the domestic fowl that are raised in Jeddah. J KAU Sci. 1992:4:87-99.

15. Hinrichsen LK, Labouriau R, Engberg RM, Knierim U, Sorensen JT. Helminth infection is associated with hen mortality in Danish organic egg production. Vet Rec. 2016; doi:10.1136/vr.103614.

16. McDougald LR. Blackhead disease (Histomoniasis) in poultry: A critical review. Avian Dis. 2005;49:462-76.
17. Eigaard NM, Schou TW, Permin A, Christensen JP, Ekstrøm CT, Ambrosini F, et al. Infection and excretion of Salmonella enteritidis in two different chicken lines with concurrent Ascaridia galli infection. Avian Pathol. 2006; 35(6):487-93.

18. Pleidrup J, Dalgaard TS, Norup LR, Permin A, Schou TW, Skovgaard K, et al. Ascaridia galli infection influences the development of both humoral and cell-mediated immunity after Newcastle disease vaccination in chickens. Vaccine. 2014;32(3):383-92.

19. CEC, Council Directive 99/74/EC: Laying down minimum standards for protection of laying hens, Official Journal of the European Communities (L 203/53). 1999;53-57. http://eur-lex.europa.eu/legal-content/EN/TXT/PDF/ ?uri=CELEX:31999L0074\&from=EN Accessed 27.Sept.2016.

20. Hinrichsen LK. Animal welfare in organic egg production- with emphasis on mortality and helminth infections. PhD thesis, Science and Technology, Department of Animal Science. Aarhus University; 2015. ISBN: 978-87-9314801-7. http://pure.au.dk/portal/files/85561971/Ph.d_71955_thesis.pdf Accessed 26.Sept.2016.

21. Wongrak K, Gauly M, Daş G. Diurnal fluctuations in nematode egg excretion in naturally and in experimentally infected chickens. Vet Parasitol. 2015;208: 195-203.

22. Daş G, Savaş T, Kaufmann F, Idris A, Abel H, Gauly M. Precision, repeatability and representative ability of faecal egg counts in Heterakis gallinarum infected chickens. Vet Parasitol. 2011;183:87-94.

23. Clarke PL. Coccidial infection with Eimeria tenella and caecal defecation in chicks. Br Poult Sci. 1979;20:317-22.

24. Klasing KC. Poultry nutrition: a comparative approach. J Appl Poultry Res. 2005;14(2):426-36.

25. Marcos-Atxutegi C, Gandolfi B, Arangüena T, Sepúlveda R, Arévalo M, Simón F. Antibody and inflammatory responses in laying hens with experimental primary infections of Ascaridia galli. Vet Parasitol. 2009;161:69-75.

26. Perez J, Zafra R, Buffoni L, Hernandez S, Camara S, Martinez-Moreno A. Cellular phenotypes in the abomasal mucosa and abomasal lymphnodes of goats infected with Haemonchus contortus. J Comp Pathol. 2008;138(2-3):102-7.

27. Kaufmann F, Daş G, Preisinger R, Schmulz M, König S, Gauly M. Genetic resistance to natural helminth infections in two chicken layer lines. Vet Parasitol. 2011:176:250-7.

28. Andersen JP, Liselotte RN, Dalgaard TS, Rothwell L, Kaiser P, Permin A, et al. No protection in chickens immunized by the oral or intra-muscular immunization route with Ascaridia galli soluble antigen. Avian Pathol. 2013; 42(3):276-82.

29. Norup LR, Dalgaard TS, Pleidrup J, Permin A, Schou TW, Jungersen G, et al. Comparison of parasite-specific immunoglobulin levels in two chicken lines during sustained infection with Ascaridia galli. Vet Parasitol. 2013;191:187-90.

30. Martín-Pacho JR, Montoya MN, Arangüena T, Toro C, Morchón R, MarcosAtxutegi C, Simón F. A coprological and serological survey for the prevalence of Ascaridia spp. in laying hens. J Vet Med B Infect Dis Vet Public Health. 2005; 52(5):238-42.

31. Sharma JM. The structure and function of the avian immune system. Acta Vet Hung. 1997:45:229-38.

32. Rose ME, Orlans E, Buttress N. Immunoglobulin classes in the hen's egg: their segregation in yolk and white. Eur J Immunol. 1974;4(7):521-3.

33. Yazwinski TA, Chapman HD, Davis RB, Letonja T, Pote L, Maes L, et al. World Association for the Advancement of Veterinary Parasitology (W.A.A.V.P.) guidelines for evaluating the effectiveness of anthelmintics in chickens and turkeys. Vet Parasitol. 2003;116:159-73.

34. Daş G, Gauly M. Density related effects on lifetime fecundity of Heterakis gallinarum in chickens. Parasit Vectors. 2014;7:334.

35. MAFF. Manual of Veterinary Parasitological Laboratory Techniques. G.D. Reference Book 418, $3^{\text {rd }}$ ed. London: Ministry of Agriculture, Fisheries and Food; 1986.

36. SAS Institute Inc. 2004. SAS/STAT ${ }^{\circledR} 9.1$ User's Guide. Cary, NC: SAS Institute Inc.

37. Swets JA. Measuring the accuracy of diagnostic systems. Science. 1988; 240(4857):1285-95.

38. SigmaPlot Version 11, from Systat Software, Inc., San Jose California USA, www.sigmaplot.com Accessed on 27 Sept 2016.

39. Solano-Gallego L, Villanueva-Saz S, Carbonell M, Trotta M, Furlanello T, Natale A. Serological diagnosis of canine leishmaniosis: comparison of three commercial ELISA tests (Leiscan ${ }^{\oplus}$, ID Screen ${ }^{\circledast}$ and Leishmania $96^{\oplus}$ ), a rapid test (Speed Leish $\mathrm{K}^{\oplus}$ ) and an in-house IFAT. Parasit Vectors. 2014;7:111.

40. Vlaminck J, Martinez-Valladares M, Dewilde S, Moens L, Tilleman K, Deforce $\mathrm{D}$, et al. Immunizing pigs with Ascaris suum haemoglobin increases the 
inflammatory response in the liver but fails to induce a protective immunity. Parasite Immunol. 2011;33(4):250-4.

41. Vlaminck J, Nejsum P, Vangroenweghe F, Thamsborg SM, Vercruysse J, Geldhof P. Evaluation of a serodiagnostic test using Ascaris suum haemoglobin for the detection of roundworm infections in pig populations. Vet Parasitol. 2012;189(2-4):267-73.

42. Püllen U, Cheat S, Moors E, Gauly M. The role of preparation technique, culture media and incubation time for embryonation of Heterakis gallinarum eggs. Dtsch Tierarztl Wochenschr. 2008;115(1):30-3.

43. Rahimian S, Gauly M, Daş G. Embryonation ability of Ascaridia galli eggs isolated from worm uteri or host faeces. Vet Parasitol. 2016;215:29-34.

44. Boes J, Nansen P, Stephenson LS. False-positive Ascaris suum egg counts in pigs. Int J Parasitol. 1997;27(7):833-8.

45. White BJ, Larson RL, Theurer ME. Interpreting statistics from published research to answer clinical and management questions. J Anim Sci. 2016;94:4959-71.

46. Permin A, Hansen JW. Epidemiology, diagnosis and control of poultry parasites. Food and Agriculture Organization of the United Nations (FAO), Animal Health Manual. No. 4. Rome. ISBN 92-5-104215-2. Available at: www. fao.org/docrep/018/x0583e/x0583e.pdf. (Accessed on 15 Nov 2016).

47. Ulmer-Franco AM, Cherian G, Quezada N, Fasenko GM, McMullen LM. Hatching egg and newly hatched chick yolk sac total IgY content at 3 broiler breeder flock ages. Poult Sci. 2012;91(3):758-64.

48. Herd RP, McNaught DJ. Arrested development and the histotropic phase of Ascaridia galli in the chicken. Int J Parasitol. 1975:5(4):401-6.

49. Luna-Olivares LA, Ferdushy T, Kyvsgaard NC, Nejsum P, Thamsborg SM, Roepstorff A, Iburg TM. Localization of Ascaridia galli larvae in the jejunum of chickens three days post-infection. Vet Parasitol. 2012;185:186-93.

50. Vatne RD, Hansen MF. Larval development of cecal worm (Heterakis gallinarum) in chickens. Poult Sci. 1965;44(4):1079-85. doi:10.3382/ps.0441079.

51. Garadaghi Y. Identification of immunogenic relevant antigens in the excretory-secretory (ES) products of Ascaridia galli larvae. Adv Environ Biol. 2011;5:1120-6.

52. Jordanova R, Radoslavov G, Fischer P, Torda A, Lottspeich F, Boteva R, et al. The highly abundant protein Ag-lbp55 from Ascaridia galli represents a novel type of lipid-binding proteins. J Biol Chem. 2005;280(50):41429-38.

53. Jeurissen SHM, Boonstra-Blom AG, Al-Garib SO, Hartog L, Koch G. Defence mechanisms against viral infection in poultry: a review. Vet Q. 2000;22:204-8.

54. Schade R, Calzado EG, Sarmiento R, Chacana PA, Porankiewicz-Asplund J, Terzolo HR. Chicken egg yolk antibodies (IgY-technology): a review of progress in production and use in research and human and veterinary medicine. Altern Lab Anim. 2005;33(2):129-54.

55. Rahimian S, Das G, Gauly M. Maternal protection against Ascaridia galli? Vet Parasitol. 2017;233:1-5. Doi: http://dx.doi.org/10.1016/j.vetpar.2016.11.014

56. Patterson R, Youngner JS, Weigle WO, Dixon FJ. The metabolism of serum proteins in the hen and chick and secretion of serum proteins by the ovary of the hen. J Gen Physiol. 1962;45:501-13.

57. Woolley JA, Landon J. Comparison of antibody production to human interleukin-6 (IL-6) by sheep and chickens. J Immunol Methods. 1995; 178(2):253-65.

58. Watson DL. Biological half-life of ovine antibody in neonatal lambs and adult sheep following passive immunization. Vet Immunol Immunopathol. 1992;30(2-3):221-32

\section{Submit your next manuscript to BioMed Central and we will help you at every step:}

- We accept pre-submission inquiries

- Our selector tool helps you to find the most relevant journal

- We provide round the clock customer support

- Convenient online submission

- Thorough peer review

- Inclusion in PubMed and all major indexing services

- Maximum visibility for your research

Submit your manuscript at www.biomedcentral.com/submit

) Biomed Central 